\title{
Integrating Ajax into GIS Web Services for Performance Enhancement
}

\author{
Seung-Jun Cha ${ }^{1}$, Yun-Young Hwang ${ }^{1}$, \\ Yoon-Seop Chang ${ }^{2}$, Kyung-Ok Kim², and Kyu-Chul Lee ${ }^{1, *}$ \\ ${ }^{1}$ Department of Computer Engineering, Chungnam National University, Korea \\ ${ }^{2}$ Electronics and Telecommunications Research Institute(ETRI), Korea \\ \{junii, yyhwang, kclee\}@cnu.ac.kr, \{ychang, kokim\}@etri.re.kr
}

\begin{abstract}
In the GIS(Geospatial Information System) Web Services, SOAP/MTOM shows best performance when transferring large size of data between services. SOAP/MTOM uses XOP for message optimizing method, so data serialization and deserialization time is reduced. Additionally, integrating Ajax(Asynchronous JavaScript and XML) approach into GIS visualization Web Services have performance enhancement, because it provides more interactive user experience. Ajax, one of the technologies of "Web 2.0", is appeared after Dot Com bubble is occurred. The intent is to make web pages feel more responsive by exchanging small amounts of data with the server behind the scenes, so that the entire web page does not have to be reloaded each time the user requests a change.
\end{abstract}

Keywords: Ajax, Web Services, GIS.

\section{Introduction}

Over the past decade GIS(Geographic Information Systems) technology has evolved from the traditional model of stand-alone systems to distributed models. Distributed GIS services will be implemented more extensively by using Web Services. But there is on additional problem: large amounts of data need to be moved among users and providers, so as to enable the first to perform their designated task. Since geographic-related data are usually large in size this exchange becomes more and more difficult despite the improvements in communication. But among SOAP message transfer methods, SOAP/MTOM[1], which is using XOP for optimizing message transfer, shows best performance when transferring large size of data between services.

The bursting of the dot-com bubble in the fall of 2001 marked a turning point for the web. The companies that had survived the collapse seemed to have some things in common. Dot-com collapse marked some kind of turning point for the web, so it is called "Web 2.0". Ajax(Asynchronous JavaScript and XML) is one of the Web 2.0's technologies. It provides asynchronous communication between the client and the

\footnotetext{
* Corresponding author.
} 
server. Integrating Ajax approach into GIS visualization Web Services have performance enhancement for user interface. [11]

This paper describe the model which is integrated Ajax into GIS Web Services, and decide how to evaluate performance between using Ajax and non-Ajax in GIS Web Services. Through the evaluation of the performance between Web Services using Ajax and Web Services using non-Ajax, results indicate integrated Ajax into Web Service model shows good performance Enhancement is approved.

The rest of this paper is organized as follows: a brief discussion of OGC GIS Web Services and Ajax is given in section 2. Section 3 shows comparisons with related work, and section 5 contains the introduction of integrated model. A definition of performance evaluation and test results are described in Section 6. Conclusions are followed in section 7 .

\section{Background}

\subsection{OGC GIS Web Services}

Geographic Information Science has a lot to benefit by the adoption of the service computing model. As mentioned also in the introduction geographic information comes from different and diverse sources and in different formats. This is especially true for the environmental related information which has to combine not only data from different sources by also models and software.[8]

Technically, Web Services technologies have provided the necessary standards for applications in different domains to integrate with GIS data and services, significant accomplishments in GIS Web Services have led to several exemplifying map and image services that adhere to Web Services standards and bring terabytes of geospatial data and digital maps to enterprise developers who house no GIS data.

The OGC(Open Geospatial Consortium) has successfully executed efforts for GIS Web Services(OWS) initiative has undergone multiple phases - including the Web Map Server(WMS), Web Feature Server(WFS), Web Coverage Server(WCS), and OGC Web Service Architecture, which support application developers in integrating a variety of online geoprocessing and location services.

\subsection{Ajax(Asynchronous JavaScript and XML)}

Ajax is a style of web application development that used a mix of modern web technologies to provide a more interactive user experience. Ajax is not a technology. It is an approach to web applications that includes a couple of technologies. These are JavaScript, HTML, Cascading Style Sheet(CSS), Document Object Model(DOM), XML and XSLT, and XMLHttpRequest as messaging protocol. [2]

These core technologies forming Ajax are mature[6], well-known and used in web applications widely. Ajax became so popular because it has a couple of advantages 
for the browser based web applications developers. It eliminates the stop-start nature of interactions, user interactions with the server happen asynchronously, data can manipulated without having to render the entire page again and again in the web browser, and requests and responses over the XMLHttpRequest protocol are structured XML documents. This enables developers easily integrate Ajax applications into Web Services.

\section{Related Works}

Previous researchers have described about applying Web Services to GIS. The paper[3] indicates about GIS Web Services about satellite imaging. It says there are problems when using GIS Web Services, because of sending large size of data. The paper[7] describes about developing GIS visualization Web Services for geophysical applications. Since images and capabilities documents can be too large and transferring these data over the internet is cumbersome, its first priority is researching technique for improving WMS performance.

Related to the Ajax, The paper[6] introduced integrating the model of Ajax approach into GIS Web Services. It describes of invoking Web Services in the Ajax Model but it is only theoretical so it has nonexistence of implementation.

Our study differs from previous studies in that we compare performance among standard SOAP, SwA/MIME, and SOAP/MTOM. So, the paper[9] indicated the result. When we send large size of data(raster and vector), SOAP/MTOM shows the best performance. And there are no studies of implementing and evaluating applications for GIS Web Services using Ajax.

\section{Integrated Ajax into GIS Web Services}

In Ajax, the client communicated with the server by using XMLHttpRequest method. XMLHttpRequest provides asynchronous access to the server simply like the Googlemap. Its performance seems better because web pages using Ajax can appear to load relatively quickly since the payload coming down is much smaller in size. So roundtrip time is less than the classic web application.

Web Services provide integrated environments within distributed system. When the client makes a request for receiving images from the server, the server runs inner JSP which can access specific Web Service of providing image files. And then the server saves them to its repository. The client's working process must be waited until response message is arrived from the server, because the client requests synchronously.

In the model integrated Web Services with Ajax (Fig. 1), as both Ajax and Web Services are used together, two different side's advantages are applied. So it can integrate Web Services from different platform's application and access to the server asynchronously. It enhances user interface and reduces roundtrip time. 


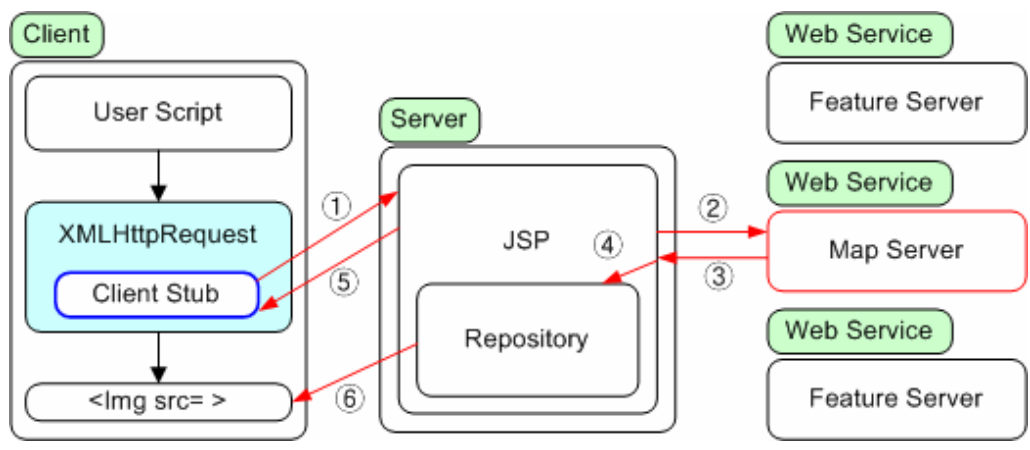

Fig. 1. Integrated Web Services with Ajax

\section{Performance Evaluation Between Using Ajax and Using Non-Ajax in GIS Web Services}

When an application is launched, images which may be displayed on the screen are fetched from the server. If the server does not have those images, the server requests for fetching images from specific Web Service. User may wait for looking displayed images for the moment. During that time, Ajax is executed, so it fetches extra images roundabout from Web Services to the server. When user drag the screen for watching another images, already fetched image is displayed faster.

\subsection{Evaluation Metrics and Method}

\subsubsection{Consideration Characteristic of Fetching Time in Ajax}

The time when user look at displayed images, the server creates a XMLHttpRequest Object and executes it. Extra images are fetched from Web Services to the server in that operation. So it takes less than user's waiting time. (Fig. 2 - (1))

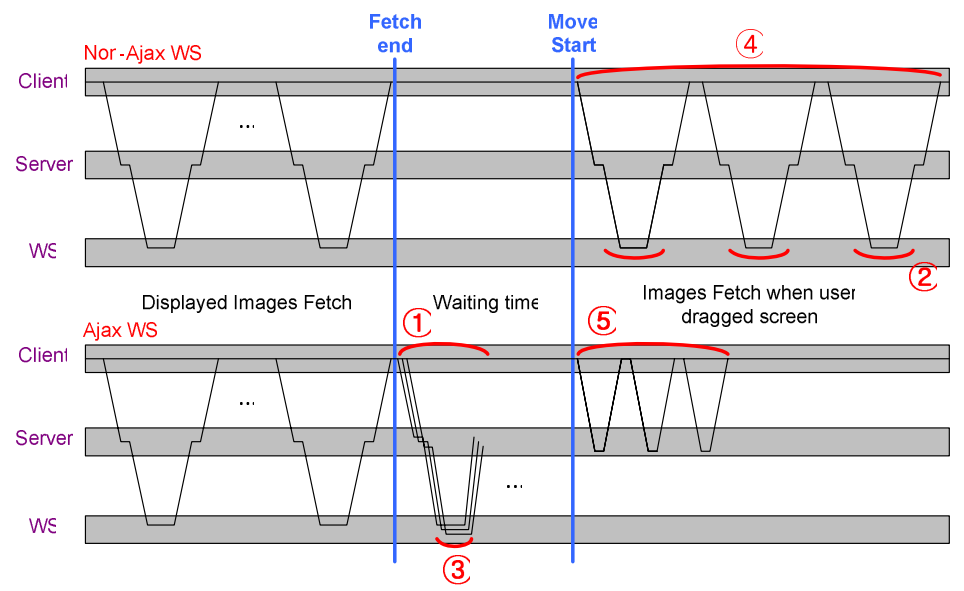

Fig. 2. Evaluation metrics 


\subsubsection{Image Fetching Time}

Displayed images must be fetched from Web Service regardless of using Ajax. Because the request using Ajax is asynchronous, images fetching time is less than using non-Ajax's fetching time. (Fig. 2 - (2), (3))

\subsubsection{User Response Time}

When users drag the screen, addition images must be fetched. Non-Ajax application is not include images in themselves, so it accesses Web Services for fetching images every time. But Ajax application has already fetched necessary images, so they are displayed faster. (Fig. 2 - (4), (5))

\subsection{Evaluation Environments and Test Data Design}

For the test, we need three computers. The configuration of the client systems is:

- Pentium 4-1.66GHz processor

- 1 Gbytes of memory

- Windows XP Pro.

The configuration of the server systems is:

- Pentium 3-1GHz *2 (Dual CPU) processors

- 2Gbytes of memory

- RedHat Linux 9

The configuration of the Web Server systems is:

- Pentium 4-1.66GHz processor

- 1Gbytes of memory

- RedHat Linux 9

All computers are connected in commonly used network. So test data is selected by considering generally used data. Google map use 20Kbytes of image tile so the tests are performed by receiving $20 \mathrm{Kbytes}$ of image.

\subsection{Evaluation Results}

The result of consideration of fetching time in Ajax is presented in Table 1. It takes almost 2 seconds while all images(12 tiles) are fetched. It shows that 2 seconds is reasonable time to fetch images while user waited.

In images fetching time, we compare Web Services using Ajax with Web Services using non-Ajax. Fig. 3 shows that if only one image is fetched, the one takes longer than the other, because it has some overheads at XMLHttpRequest method. But, if multiple images are fetched the other takes longer than the one, because Ajax can communicate asynchronously.

Table 1. Consideration characteristic of fetching time

\begin{tabular}{cccccc}
\hline & 1 & 3 & 6 & 9 & 12 \\
\hline Ajax & $186 \mathrm{~ms}$ & $475 \mathrm{~ms}$ & $938 \mathrm{~ms}$ & $1410 \mathrm{~ms}$ & $1988 \mathrm{~ms}$ \\
\hline
\end{tabular}




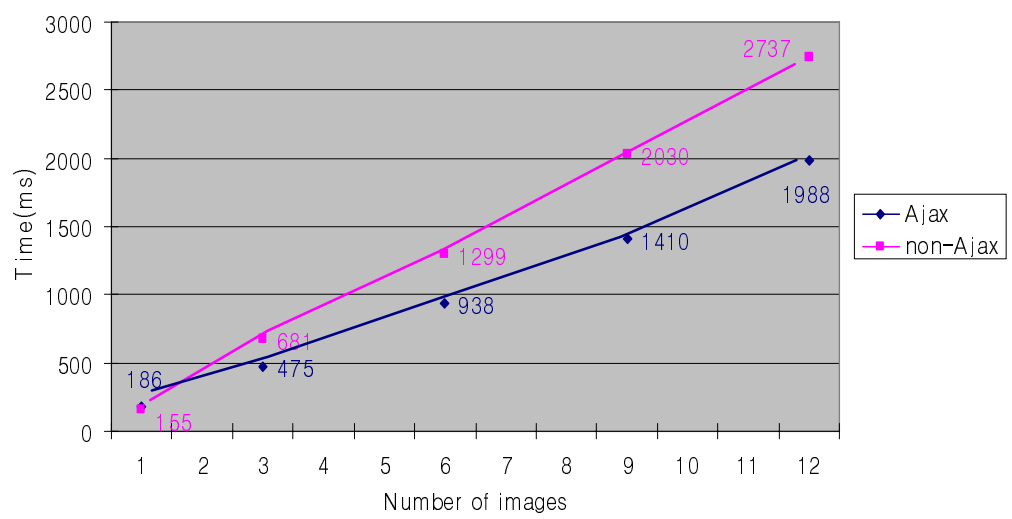

Fig. 3. Image fetching time

Fig. 4 indicated the user roundtrip time. In Web Services using Ajax the server has already read extra images and save them to its repository. So when the client requests mages, it can read them directly and the server does not need to connect Web Services. However in non-Ajax application, when the client requests images the server must connect Web Services for fetching images every time. So it takes longer than before. The roundtrip time is most important factor for user interface. If roundtrip time measured less, users realize that the application has good performance. The more evaluation data comes to many, the more gaps we get.

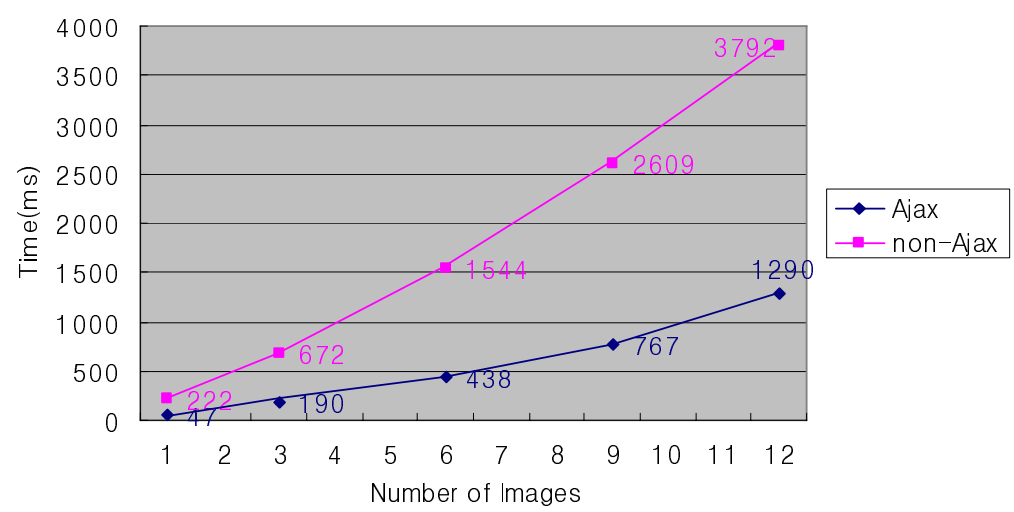

Fig. 4. User response time

\section{Conclusion}

Our research is for performance enhancement in GIS Web Services. So we have performed evaluation experiments of SOAP and its variants for the transmission efficiency. Our tests indicate that SOAP performance is improved when using SOAP/MTOM for sending protocol, in both raster data and vector data. It is because 
SOAP/MTOM uses XOP optimizing mechanism so that serialization time and deserialization time is reduced. Especially in vector data, a standard SOAP message is made by embedding vector data as elements or attributes. Although total message size using SwA/MIME and SOAP/MTOM is bigger than standard SOAP because of tagging, roundtrip time is less than that. It is because SwA/MIME and SOAP/MTOM consist of small SOAP message and attachments exist in boundaries.

In addition to precede test, GIS Web Services' performance may have enhancement by using asynchronous technology. So we have chosen Ajax technology, one of the Web 2.0 technologies. Ajax provides asynchronous communication when the client request required images to the server. Web Services and Web Services using Ajax comparison results that Web Services using Ajax represent good performance in images fetching and user roundtrip time because it fetches them beforehand.

These results will contribute to integration construction's main technology of GIS systems. And also they will also contribute that in Web Services of dealing with large size of data used in GIS system, its performance should be enhanced.

\section{Acknowledgement}

This research was supported by the Ministry of Information and Communication, Korea, under the College Information Technology Research Center Support Program, grant number IITA-2006-C1090-0603-0031.

\section{References}

1. Gudgin, M., et al., 2005, SOAP Message Transmission Optimization Mechanism, (on-line) http://www.w3.org/TR/soap12-mtom/

2. Jesse James Garret, Ajax: A New Approach to Web Applications. 2005. 2, http:// www.adaptivepath.com/ publications/essays/archives/000385.php

3. Kotzinos, D., et al., GIS Web Services and Satellite Imaging as a Framework for Environmental Monitoring: The Design and Implementation of a Vegetation Indices Calculation Test Case, Computer Science Department, University of Crete, Greece

4. Mitra, N., 2003, SOAP Version 1.2 Part 0:Primer, (on-line) http:// www.w3.org/ TR/ 2003/ soap12-part0/

5. Ng, A., et al., 2005, A Study of the Impact of Compression and Binary Encoding on SOAP performance, Department of Computing, Macquarie University, Australia

6. Sayar, A., et al., 2006, Integrating AJAX Approach into GIS Visualization Web Services, Community Grids Lab, Indiana University, Bloomington, Indiana

7. Sayar, A., et al., Developing GIS Visualization Web Services for Geophysical Applications, Community Grids Lab, Indiana University, Bloomington, Indiana

8. Tu, S., et al., 2006, Web Services for Geographic Information System, IEEE internet Computing, pp. 13-15

9. Seung-Jun, C., et al., A Performance Evaluation of SOAP Variants for GIS Web Services, ISRS 2006 PORSEC, Vol 2(2006), 615-618

10. Tim O'Reilly, What Is Web 2.0. Design Pattern and Business Models for the Next Generation of Software, O'REILLY, 2005. 9

11. Ying, Y., et al., 2004, A performance Evaluation of Using SOAP with Attachments for eScience, School of Computer Science, Cardiff University1.1 Checking the PDF File 